\title{
Single- or multiple-visit endodontics: which technique results in fewest postoperative problems?
}

\author{
Abstracted from \\ Sathorn C, Parashos P, Messer H. \\ The prevalence of postoperative pain and flare-up in single- and multiple-visit endodontic treatment: \\ a systematic review. Int Endod J 2008; 41: 91-99 \\ Address for correspondence: Dr. Chankhrit Sathorn, 720 Swanston Street, Melbourne, Victoria 3010, \\ Australia. E-mail: sathornc@unimelb.edu.au
}

\section{Question: When individuals undergo root canal treatment, does a single-visit approach result in a higher frequency of post-treatment problems than a multiple-visit one?}

Data Sources The Cochrane Central Register of Controlled Trials, Medline, Embase, six thesis databases (Networked Digital Library of Theses and Dissertations, Proquest Digital Dissertations, OAlster, Index to Theses, Australian Digital Thesis Program and Dissertation.com) and one conference report database (BIOSIS Previews) were searched. There were no language restrictions.

Study selection Studies were included if subjects had a noncontributory medical history; underwent nonsurgical root canal treatment during the study; there was comparison between single- and multiple-visit root canal treatment; and if outcome was measured in terms of pain degree or prevalence of flare-up.

Data extraction and synthesis Data were extracted using a standard data extraction sheet. Because of variations in recorded outcomes and methodological and clinical heterogeneity, a meta-analysis was not carried out, although a qualitative synthesis was presented.

Results Sixteen studies fitted the inclusion criteria in the review, with sample size varying from 60-1012 cases. The prevalence of postoperative pain ranged from $3-58 \%$. The heterogeneity of the included studies was far too great to yield meaningful results from a meta-analysis. Conclusions Compelling evidence is lacking to indicate any significantly different prevalence of postoperative pain or flare-up following either single- or multiple-visit root canal treatment.

\section{Commentary}

Historically, root canal treatment was performed over multiple visits. This was primarily to ensure 'sterility' of the root canal system prior to obturation and to allow enough operational time to achieve the mechanical and biological objectives of the treatment. Modern advances in the theory and practice in endodontics inform us, beyond any doubt, that the theory of sterility is not achievable clinically. Meanwhile, the development of innovative instruments and devices has increased the efficiency of treatment performance. Practitioners who believe that successful and painless root canal treatment can be accomplished in one visit and those who prefer multiple-visit procedures can all find a rationale in the literature. ${ }^{1-3}$

Our knowledge of the pathogenesis of these complications indicates that the causative factors of flare-ups comprise mechanical, chemical and/ or microbial injury to the periapical tissues. ${ }^{1}$ Indeed, most cases of flare-up occur as a result of acute periapical inflammation, secondary to intra-canal procedures. Regardless of the type of injury, the intensity of the inflammatory response is directly proportional to the intensity of the tissue injury, which results in a cascade of cellular and molecular events ending in the clinical scenario of flare-ups.

The heterogeneity encountered in the present systematic review reveals the enormous variables involved in trying to address the questions of the study. The conclusion is not surprising at all, then, considering the proposed mechanisms of pathogenesis of this pathophysiological phenomenon. One might assume that incidence of post-operative flare-ups following use of the traditional treatment approaches and armamentarium would be different from that with current approaches. For example, the use of rotary nickel-titanium files result in less debris extrusion to the periapical area compared with the step-back technique, and thus provokes fewer postoperative complications. Another variable is the technique, volume and concentration of intra-canal irrigation, which directly impact on the microbial load of the root canal system and elsewhere.

The results of this review highlight the need for well-controlled clinical research to establish the factors associated with successful implementation of single-visit treatment and re-treatments.

\section{Khaled Balto}

Faculty of Dentistry, King Abdulaziz University, Jeddah, Saudi Arabia

1. Seltzer S, Naidorf IJ. Flare-ups in endodontics. Part I. Etiological factors. J Endodont 1985; 11: 472-478.

2. Siqueira Jr JF, Rocas IN, Favieri A, et al. Incidence of post-operative pain following intracanal procedures based on an antimicrobial strategy. J Endodont 2002; 28: $457-460$

3. Wolton R, Fouad A. Endodontic inter-appointment flare-ups a prospective study of incidence and related factors. J Endodont 2002; 18: 172-177.

Evidence-Based Dentistry (2009) 10, 16. doi:10.1038/sj.ebd.6400630 